\title{
LOGÍSTICA REVERSA COMO FORMA DE DESENVOLVIMENTO SUSTENTÁVEL E COMPETITIVO DAS EMPRESAS
}

\author{
REVERSE LOGISTICS AS A WAY OF DEVELOPING \\ SUSTAINABLE AND COMPETITIVE BUSINESS
}

\author{
Mayara Cristina Ghedini da Silva ${ }^{1}$, João Carlos Colmenero ${ }^{2}$ \\ ${ }^{1}$ UTFPR- Programa de Pós-Graduação em Engenharia de Produção - \\ E-mail: <mayara_ghedini@hotmail.com> \\ ${ }^{2}$ UTFPR- Programa de Pós-Graduação em Engenharia de Produção - \\ E-mail: <j-colmenero@utfpr.edu.br>
}

\section{RESUMO}

A Logística Reversa (LR) consiste em uma atividade responsável pelo retorno dos materiais do ponto de consumo ao ponto de origem, agregando valor de diferentes naturezas ao produto retornado. Este trabalho procura evidenciar a LR como uma atividade que apresenta um diferencial competitivo e redução de custos por meio das formas de gestão e desenvolvimento que envolvem a atividade. A metodologia utilizada para a elaboração desta pesquisa é considerada exploratória e descritiva. Classifica-se como descritiva por apresentar uma revisão bibliográfica, em consulta a livros sites da Internet, artigos e trabalhos acadêmicos, buscando conceitos e informações para a elaboração de uma base conceitual sobre o tema proposto. A pesquisa apresenta a LR como uma atividade que agrega valor sustentável e financeiro, devido ao aumento da preocupação dos clientes com o meio ambiente.

Palavras-Chave: Logística reversa. Valor financeiro. Preocupação ambiental.

\begin{abstract}
Reverse Logistics (RL) consists of an activity responsible for the return of materials from the point of consumption to the point of origin, adding different value to the product returned. This paper emphasizes LR as an activity that has a competitive advantage and cost reduction through the forms of management and development involved in the activity. The methodology used in the research is considered exploratory and descriptive. It is classified as descriptive because it presents a literature review that included books, web sites, articles and papers,
\end{abstract}


looking for concepts and information for the elaboration of a conceptual basis on the proposed topic. The research presents $t$ LR as an activity that adds sustainable and financial value due to increase of customers' concern with the environment.

Keywords: Reverse logistics. Financial value. Environmental concern.

\section{INTRODUÇÃO}

A partir da Revolução Industrial, as fábricas começaram a produzir objetos de consumo em larga escala, a grande maioria das indústrias consideravam o meio ambiente como um local somente para obtenção de matéria-prima e descarte dos resíduos. Os resultados do crescimento econômico mundial causam impactos de degradação ambiental sem precedentes das atividades produtivas.

Segundo Fuller et al. (1995), atualmente, muitas vezes o lixo é tratado com a mesma indiferença da época das cavernas, quando o mesmo não era verdadeiramente um problema, seja pela menor quantidade gerada, ou seja pela maior facilidade da natureza em reciclá-lo. Entretanto, atualmente, a quantidade de lixo gerada no mundo tem sido grande, e seu gerenciamento inadequado, além de provocar gastos financeiros significativos, pode provocar graves danos ao meio ambiente, comprometer a saúde e o bem-estar da população.

As primeiras indústrias surgiram numa época em que não existia a preocupação com os problemas ambientais, principalmente pela diminuta escala de produção, a fumaça era sinal de progresso e desenvolvimento de um país, usada como propaganda e símbolo de desenvolvimento por alguns governantes (DONAIRE, 1999).

Saliente-se que a Conferência de Estocolmo, em 1972, foi o marco inicial da preocupação com a preservação e cuidado com o meio ambiente. $\mathrm{O}$ evento foi o primeiro encontro de representantes de diversos países, para discutirem os problemas ambientais que estavam começando a ocorrer.

Kraemer (2006) explica que a preocupação mundial em torno do meio ambiente caminha para um consenso em torno da adesão a um novo estilo de desenvolvimento que deve combinar eficiência econômica com a justiça social e prudência ecológica.

A LR é a atividade que se preocupa em reaproveitar, reciclar, reutilizar ou dar a destinação final adequada a materiais, componentes e resíduos industriais, agregando-lhes valores de diferentes naturezas.

O processo LR divide-se em duas áreas: pós-venda e pós-consumo. São denominados bens de pós-venda, produtos sem ou com pouco uso, que retornam as lojas/fábricas por motivos de acordos comerciais estabelecidos previamente entre as partes, para substituição de componentes, entre outros. Esta é uma área da logística muito aplicada nas empresas/lojas, porém, nem sempre denominada como uma atividade LR.

São classificados bens de pós-consumo materiais em final de vida útil ou que ainda apresentam condições de serem reutilizados, e resíduos industriais. Estes bens são caracterizados por ter a possibilidade de dar origem a um novo produto, ou pela reciclagem do material, ou pela reutilização de componentes.

A implantação do processo LR é estimulada por fatores que agregam valor ao produto retornado. Estes fatores preocupam-se com questões ambientais, aumento da competitividade empresarial, redução de custos, legislações, entre outras.

As atividades da LR são incentivadas pela sociedade que se demonstram cada vez mais preocupadas com questões ecológicas e em consumir produtos que não agridam o meio ambiente. Assim faz com que empresários e fabricantes procurem adequar-se a essas exigências adaptando o processo produtivo para obter produtos ecologicamente corretos. Deste modo, as empresas minimizam riscos de multas ambientais, aumentando assim, a possibilidade de lucros, por vender produtos que agregam valor ecológico.

\section{LOGÍSTICA REVERSA}

Muitas vezes a LR é relacionada apenas como uma atividade que envolve questões ambientais e ecológicas, devido ao fato da reciclagem ser um dos temas norteadores do processo. 
Entretanto, Felizardo (2003) explica que o objetivo da LR é agregar valor a um produto que possui componentes em condição ou não de uso, resíduos industriais e produtos que apresentam fim de sua vida útil.

$\mathrm{Na}$ atualidade, a implantação do processo logístico reverso pode evitar ou amenizar os impactos ambientais. A preocupação com o consumo sustentável está sendo influenciadas devido as legislações ambientais cada vez mais rígidas, onde os fabricantes são responsáveis por seus produtos durante toda a vida útil do mesmo, e também pelos resíduos gerados no processo produtivo (DAHER; SILVA; FONSECA, 2006).

A carência de informações faz com que haja dificuldade na visualização dos custos. Porém, economias podem ser obtidas, como na utilização de embalagens retornáveis e no reaproveitamento de materiais para a produção (RODRIGUES et al., 2002).

Para que isso ocorra, faz-se necessário entender que a vida útil de um produto é contabilizada a partir do momento de sua produção até ser adquirido por um consumidor. A vida útil pode ser aumentada desde que exista a possibilidade de aumentar sua utilização por meio de uma nova inserção na cadeia de consumo (LEITE, 2003). De acordo com o autor, os bens são classificados de acordo com sua vida útil em:

- Bens duráveis: bens que possuem vida útil que varia de alguns anos e algumas décadas;

- Bens semiduráveis: materiais que possuem uma vida útil intermediária, entre durável e descartável, sua vida corresponde a meses, dificilmente é superior a dois anos;

- Bens descartáveis: bens que tem vida útil de descartes de apenas algumas semanas, raramente passam de seis meses.

Segundo Stock (2001a) para uma empresa destaque-se frente às demais, é importante que desenvolva atividades na área da LR. Devido ao fato desta, estar diretamente relacionado a questões ecológicas, legais e econômicas, e Pires (2007) afirma ser imprescindível o seu estudo no atual contexto organizacional.
A LR no Brasil pode ser vista de dois ângulos: está defasada em relação aos padrões dos países desenvolvidos, entretanto, está apresentando mudanças positivas e grandes perspectivas (SINNECHER, 2007). Fleury et al. (2000) afirma que a partir da década de 90, a LR brasileira passou por várias mudanças, porém, o processo inflacionário é visto como um dos maiores motivos para o atraso no desenvolvimento do processo.

\subsection{Importância da LR}

A importância da LR pode ser vista em dois âmbitos, sendo: o econômico e o social. O econômico diz respeito aos gastos financeiros obtidos por meio das práticas que envolvem a atividade. E o social refere-se aos ganhos recebidos pela sociedade (CAMPOS, 2006).

Simões (2002) afirma que com o aumento de práticas e atividades ambientais, pode ser visualizada a importância da LR, e a atividade passou a ser considerada uma forma de unir todo o ciclo da cadeia de suprimentos.

O processo logístico reverso, para Guarnieri et al. (2006), tornou-se um assunto de grande importância atualmente, por diferentes motivos, como: redução do ciclo de vida e a obsolescência precoce dos produtos; criação de novas tecnologias e de produtos constituintes de novos materiais; entre outras.

Biazzi (2002) ressalta algumas razões que demonstram a importância do processo Logístico Reverso:

- Devolução cada vez maior de produtos pelos clientes do varejo;

- Alto desenvolvimento tecnológico, o que provoca uma grande obsolescência e aumenta a preocupação das empresas em evitar acúmulos de produtos;

- Escassez de recursos virgens;

- Dificuldade de eliminar produtos e componentes não reaproveitados nas grandes cidades, gerando acúmulo de resíduos.

A LR contempla diferentes atividades, não apenas o retorno de produtos ao fabricante/processo produtivo. Campos (2006) explica que a LR preocupa-se com razões de retorno de produtos pós-venda: inconformidade, defeito, não atendimento das expectativas dos clientes. E no pós-consumo: 
obsolescência ou final da vida útil e destinação correta dos produtos.

$\mathrm{Na}$ atualidade, empresas que adotarem o processo logístico reverso ganham tanto no fortalecimento de sua imagem institucional, o que possibilita uma nova gama de negócios, a geração de empregos, de serviços e o desenvolvimento tecnológico (FELIZARDO; HATAKEYAMA, 2002).

\subsection{Formas de gestão da LR}

As formas de gestão da LR são descritas em suas principais áreas de descarte: Reutilização; Desmanche; Venda ao mercado secundário; Remanufatura; Reciclagem e Disposição final.

\subsubsection{Reutilização}

Reutilizar significa, segundo Barbieri (2004), utilizar os resíduos do mesmo modo em que foram produzidos, como por exemplo: re-trabalhar peças; reaproveitamento de restos de matérias-primas; prolongamento da vida útil de pallets, tambores e embalagens, entre outras.

Esta é considerada por Leite (2003) uma atividade de grande importância, pois possibilita a distribuição dos bens em subcanais reversos como: o desmanche de bens, componentes ou sucatas para a indústria responsável pela transformação dos materiais.

\subsubsection{Desmanche}

O processo de desmanche de matérias tem como objetivo, que de maneira fácil e rápida, as peças e componentes sejam aproveitados, de uma maneira economicamente sustentável (MOURA, 2002).

Segundo Leite (2003), o canal reverso de desmanche é uma forma de revalorizar um produto durável pós-consumo. $\mathrm{O}$ autor explica que nesta etapa o produto passa por um processo de desmontagem, no qual são retirados os componentes que apresentam condição de serem usados e/ou revalorizados.

Este mesmo autor complementa informando que por meio do desmanche é possível revalorizar os componentes destinados ao mercado secundário ou remanufaturados para serem comercializados no mercado primários. Isso porque, os materiais que não podem ser reaproveitados podem ser comercializados em empresas de reciclagem industrial ou encaminhados à destinação final.

\subsubsection{Venda ao mercado secundário}

O mercado secundário pode ser considerado uma maneira de revalorização de produtos, desde que os mesmos apresentem condições de uso e que haja interesse por parte dos consumidores em utiliza-los (HASS; MONTEIRO, 2008).

Para Leite (2003) o mercado secundário se apresenta como uma parcela importante na economia que a atividade reversa da proporcionada na sociedade, entretanto seus valores se apresentam pouco documentados.

\subsubsection{Remanufatura}

O processo de remanufatura consiste na atividade de desmontagem, limpeza, conserto e substituição das peças danificadas, finalizando com a montagem do produto. As peças remanufaturadas, podem ser colocadas no mesmo ou em outro produto, desempenhando sua função original ou uma nova (GRAEDEL; ALLENBY apud FRANCISCO JUNIOR et al., 2003).

Para Costa Filho et al. (2006) a indústria de remanufatura está em expansão e surge como uma alternativa para as empresas, pois estimula a durabilidade do produto, a redução de custos e a minimização de impactos ambientais.

\subsubsection{Reciclagem}

"Entende-se por reciclagem o processo em que componentes de produtos já usados sofrem transformação de forma que a matéria-prima neles contida possa ser reincorporada à fabricação de novos produtos" (NOVAES, 2007).

Leite (2003) contribui com o conceito anteriormente apresentado, informando que a reciclagem é uma atividade de revalorização que extrai industrialmente os componentes dos produtos que foram descartados, e que são incorporados à fabricação de novos produtos.

A reciclagem pode ser considerada um avanço tecnológico, pois nesta operação os produtos são reprocessados e reaproveitados. O processo de reciclagem, em alguns casos, possui um custo elevado, 
porém, contribui para a proteção do meio ambiente, por meio da redução do lixo (RIBEIRO, 2005).

\subsubsection{Incinerar}

Segundo Leite (2003) o processo de incineração pode agregar valor econômico aos resíduos, pois transforma o vapor em energia elétrica, entretanto, essa atividade gera gases nocivos ao meio ambiente.

Moura (2002) informa que a incineração gera uma quantidade grande de cinzas, quantidade esta que chega ser maior que a queima de carvão, que por sinal gera muito mais energia, o autor complementa dizendo que "é preciso muito cuidado no manuseio dessas cinzas, que podem conter elementos carcinogênicos".

\subsubsection{Disposição final}

Etapa na qual os produtos descartados e não possuem mais utilidade para o processo industrial, existe a preocupação e a necessidade de garantir uma disposição final adequada aos produtos, de modo a serem dispostos evitando poluição e agressão ao meio ambiente (NOVAES, 2007).

Leite (2003) explica que esta é a última etapa, na qual os produtos que não possuem condições de serem revalorizados são destinados. $\mathrm{O}$ autor ainda informa que a disposição final não controlada, consiste na disposição de resíduos em lixões, córregos, rios e terrenos, provocando assim, uma poluição ambiental.

\section{da LR \\ 2.3 Fatores que motivam a implantação}

Os fatores que motivam a implantação do processo logístico reverso variam em função do tipo de produto e de retorno que ocorrem no processo logístico reverso de pós-venda e pós-consumo.

\subsubsection{Sensibilidade ecológica}

O aumento da descartabilidade dos produtos após seu primeiro uso vem provocando o desequilíbrio entre quantidade descartada e a reaproveitada, gerando um grande crescimento de produtos de pós-consumo. Ocorrendo assim, dificuldade de disposição do lixo urbano (LEITE, 2003).
A LR oferece benefícios para a empresa que a utiliza e para a sociedade, já que por meio do gerenciamento do processo reduz custos e minimiza impactos ambientais (BISPO, 2007).

A quantidade excedente de produtos que são descartados de forma errada tornou-se uma grande preocupação. Leite (2003) explica que a Sensibilidade Ecológica gerada da preocupação com o meio ambiente, "tem se tornado um importante fator de incentivo à estruturação e à organização dos canais de distribuição reverso".

Cury; Petkow e Grande (2003) complementa a afirmação anterior, informando que a LR pode ser considerada uma ferramenta de grande valia, para uma proposta de produção e consumo sustentável.

O autor complementa informando que a Sensibilidade Ecológica tem sido acompanhada por ações de empresas e governos, com o objetivo de diminuir os efeitos visíveis dos diferentes tipos de impactos ambientais, protegendo assim, a sociedade e seus próprios interesses.

\subsubsection{Competitividade empresarial}

O nível de competitividade vem aumentando, de acordo com o crescimento do número de empresas que oferecem os mesmos produtos ou serviços, o que consequentemente faz com que os clientes exijam mais qualidade e maiores benefícios para adquiri-los.

Giacobo, Estrada e Ceretta (2003) explicam que é muito importante implantar as atividades da LR de forma clara e objetiva, para que todos os envolvidos na atividade considerem o processo como uma fonte potencial de vantagem competitiva, e não apenas, um centro de custos para a empresa.

Para Resende (2004) a LR pode ser usada para manter a fidelidade dos clientes com seu fornecedor. $\mathrm{O}$ autor exemplifica dizendo que ao facilitar de forma rápida o retorno e a substituição de um produto que apresentou defeito, o fornecedor cativa, e dificulta o afastamento deste cliente.

Leite (2003) explica que as empresas líderes aplicam a LR por meio de métodos de consignação de mercadorias, liquidação ou limpeza de estoques nos canais, recepção de devoluções por motivos de qualidade, garantia de pós-venda ou de validade do produto, entre outros. 
O gerenciamento do processo logístico reverso é custoso, entretanto aumenta o prestígio da empresa frente à sociedade. Devido a isso, a LR pode ser utilizada como ferramenta de estratégia de marketing, se tornando um fator de Competitividade Empresarial.

\subsubsection{Diferenciação da imagem corporativa}

A preocupação com o retorno de produtos, pós-venda e/ou pós-consumo, aliada a utilização do marketing ambiental, proporciona a visualização de uma empresa ecologicamente correta, agregando assim uma diferenciação da imagem corporativa (BRAGA JUNIOR, 2007).

Para Leite (2003) as empresas procuram posicionar sua imagem frente a questões ambientais, devido ao fato das ações dirigidas a preservação ambiental, proporcionarem grandes retornos de imagem diferenciada gerando vantagem competitiva.

A LR pode oferecer as empresas que a utilizam uma imagem de preocupação com as pessoas e com a comunidade que está inserida. Deste modo, agregam valor a sua marca e aos seus produtos (BRAGA JUNIOR, 2007).

As empresas podem ser ecologicamente corretas por meio de políticas de preservação do meio ambiente em seu dia-a-dia, e participando de projetos sociais que tem por objetivo desenvolver a comunidade de forma organizada, reduzindo os problemas sociais da região (RODRIGUES et al., 2002).

\section{METODOLOGIA}

O presente trabalho é classificado como: exploratório e descritivo, do ponto de vista de seus objetivos, por meio do método indutivo. Considera-se uma pesquisa exploratória pelo fato de promover maior conhecimento do tema proposto, por meio de pesquisas bibliográficas e estudo de caso (GIL, 1996).

Também é uma pesquisa descritiva, por apresentar características de um ambiente produtivo com o qual o modelo é comparado. A pesquisa descritiva tem por objetivo descrever características de uma população ou fenômeno ou estabelecer relações entre variáveis. E a pesquisa envolve o uso de técnicas de coletas de dados (GIL apud SILVA; MENEZES, 2001).
Um trabalho de revisão bibliográfica buscando um embasamento teórico sobre o tema pesquisado. Para Gil (2002) este é um método que permite o estudo de uma ampla gama de atividades e conceitos. Com tudo, foram realizadas coletas de informações a respeito da LR e seus componentes, por meio de consulta em livros, trabalhos acadêmicos e sites na Internet, consolidando-se informações e conhecimentos sobre o tema, para se apresentar de forma clara e objetiva o tema pesquisado.

\section{CONCLUSÃO}

Este trabalho apresenta uma estrutura conceitual da atividade LR, buscando explicitar o que é a atividade, quais suas formas de gestão e os fatores que motivam a implantação e o gerenciamento da atividade, onde se busca agregar valor de diferentes naturezas aos produtos retornados.

Conclui-se que a LR é constantemente relacionada a questões ecológicas e ambientais, devido ao fato de estar diretamente relacionada a atividades de reciclagem e reutilização. Entretanto, seu objetivo é aliar o desenvolvimento sustentável, o diferencial competitivo e razões financeiras de uma empresa.

A reciclagem, o retrabalho e a reutilização de produtos estão proporcionando um diferencial competitivo as empresas que aderem a estas atividades. Este fator vem ocorrendo, devido ao aumento da preocupação e da exigência dos clientes com o meio ambiente, favorecendo assim, a implantação do processo.

\section{REFERÊNCIAS}

BARBIERI, J. C. Gestão ambiental empresarial: conceitos, modelos e instrumentos. São Paulo: Saraiva, 2004.

BIAZZI, L. F. Logística reversa: o que é realmente e como é gerenciada. São Paulo: USP, 2002.

BISPO, E. C. Logística reversa: benefícios e contribuições para a sociedade e meio ambiente no caso da garrafa de água mineral de 20 litros em uma empresa do ramo. Faculdade de Ciência e Tecnologia da Bahia, Salvador, 2007.

BRAGA JUNIOR, S. S. Gestão ambiental no varejo: um estudo das práticas de logística reversa em supermercados de médio porte. 2007. 133 f. Dissertação (Mestrado em 
Administração das Organizações) - Programa de PósGraduação em Administração das Organizações, Faculdade de Economia, Administração e Contabilidade de Ribeirão Preto, Ribeirão Preto, 2007.

CAMPOS, T. Logística reversa: aplicação ao problema das embalagens da CEAGESP. 2006. 168 f. Dissertação (Mestrado em Engenharia) - Escola Politécnica, Universidade de São Paulo, São Paulo, 2006.

COSTA FILHO, C. F. F.; COELHO JUNIOR L. C. B.; COSTA, M. G. F. Indústria de cartucho de toner sob a ótica da remanufatura: estudo de caso de um processo de melhoria. Revista Produção, v. 16, n. 1, p. 100-110, Jan./ Abr. 2006. Disponível em: <http://www.scielo.br/pdf/prod/ v16n1/a09v16n1.pdf $>$. Acesso em: outubro de 2009.

CURY, R. M.; PETKOW, M.; GRANDE, M. B. de. Logística reversa na hotelaria: estudo de caso em hotel certificado pela ISO 14001. In: ENCONTRO NACIONAL DE ENGENHARIA DE PRODUÇÃO, 23. Ouro Preto MG, 2003.

DAHER, C. E.; SILVA, E. P. L. S.; FONSECA, A. P. Logística reversa: oportunidade para redução de custos através do gerenciamento da cadeia integrada de valor. In: CONGRESO INTERNACIONAL DE COSTOS, 8, 2003, Punta del Este. Nuevos desafíos de la gestión empresarial ante un mundo globalizado y competitivo, Punta del Este, 2003.

DONAIRE, D. Gestão ambiental na empresa. 2 ed. São Paulo: Atlas, 1999.

FELIZARDO, J. M.; HATAKEYAMA, K.. Logística reversa como agente cibernético. In: ENCONTRO NACIONAL DE ENGENHARIA DE PRODUÇÃO, 22. Curitiba, 2002.

FELIZARDO, J. M. Logística reversa: a reciclagem nas industrias de plástico da cidade de Curitiba e circunvizinhas. 2003. 151 f. Dissertação (Mestrado em Tecnologia) Programa de Pós-Graduação em Tecnologia. Centro Federal Tecnológico de Educação Tecnológica do Paraná, Curitiba, 2003.

FLEURY, P. F.; FIGUEIREDO, K. F.; WANKE, P. Logística empresarial: a perspectiva Brasileira. Coleção COPPEAD de Administração. São Paulo: Atlas, 2000.

FRANCISCO JUNIOR, M.; GIANNETI, B. F.; ALMEIDA, C. M. V. B. Ecologia industrial: projeto para meio ambiente. Revista de Graduação da Engenharia Química, ano 6, n.12, jul-dez/2003. Disponível em: <http://www.hottopos. com/regeq12/art5.htm> Acesso em: novembro de 2009.

FULLER, D.A.; ALLEN, J. Reverse Channel Systems. Environmental marketing: strategies, practice, theory and research. London: The Haworth Press 1995.

GIACOBO, F.; ESTRADA, R. S.; CERETTA, P. S. Logística reversa: a satisfação do cliente no pós-venda.
Revista Eletrônica de Administração - READ/UFRGS, Porto Alegre, v. 9, n. 5, p. 01-17, set-out/2003. Disponível em: < http://read.adm.ufrgs.br/edicoes/busca.php>. Acesso em: novembro de 2009.

GIL, A. C. Como elaborar projetos de pesquisa. São Paulo: Atlas, 2002. . São Paulo: Atlas, 1996.

GUARNIERI, P. Obtendo competitividade através da logística reversa: um estudo de caso em uma madeireira. Journal of Technology Management \& Innovation, v. 1, p. 121-130, 2006.

HASS, D.; MONTEIRO, G. T. S. A mensuração da logística reversa através da contabilidade ambiental em uma empresa do ramo de transportes da Região dos Campos Gerais. 2008. 198 f. Relatório final de estágio (Bacharel em Ciências Contábeis) - Departamento de Contabilidade, Universidade Estadual de Ponta Grossa, Ponta Grossa, 2008.

KRAEMER, M. E. P. Contabilidade ambiental: Relatório para um futuro sustentável, responsável e transparente. Universo Ambiental, São Paulo, 2006. Disponível em: $<$ http://www.universoambiental.com.br/Contabilidade/ Contabilidade_FuturoSust.htm $>$ Acesso em: outubro de 2009.

LEITE, P. R. Logística reversa: meio ambiente e competitividade. São Paulo: Pearson Prentice Hall, 2003.

MOURA, L. A. A. Qualidade e gestão ambiental. 3. ed. São Paulo: Juarez de Oliveira, 2002.

NOVAES, A. G. Logística e gerenciamento da cadeia de distribuição. 3. ed. Rio de Janeiro: Elsevier, 2007.

RESENDE, E. L. Canal de distribuição reverso na reciclagem de pneus: estudo de caso. 2004. $120 \mathrm{f}$. Dissertação (Mestrado em Engenharia Industrial) Programa de Pós-Graduação em Engenharia Industrial, Pontifícia Universidade Católica do Rio, Rio de Janeiro, 2004.

RIBEIRO, M. S. Contabilidade ambiental. São Paulo: Saraiva, 2005.

RODRIGUES, D. F.; RODRIGUES, G. G.; LEAL, J. E.; PIZZALOTO, N. D. Logística Reversa - conceitos e componentes do sistema. In: ENCONTRO NACIONAL DE ENGENHARIA DE PRODUÇÃO, 22. Curitiba, 2002.

ROGERS, D. S., TIBBEN-LEMBKE. Going backwards: reverse logistics trends and practices. Reno, University of Nevada, Reverse Logistics Executive Council: 1999.

SILVA, E. L.; MENEZES, E. M. Metodologia da pesquisa e elaboração de dissertação. 3.ed. Florianópolis: Laboratório de Ensino à Distância da Universidade Federal de Santa Catarina, 2001. 
SIMÕES, J. C. P. A logística reversa aplicada à exploração e produção de petróleo. Dissertação (Mestrado em Engenharia de Produção) - Programa de Pós-Graduação em Engenharia de Produção, Universidade Federal de Santa Catarina, Florianópolis, 2002.

SINNECHER, C. A. Estudo sobre a importância da logística reversa em quatro grandes empresas da Região Metropolitana de Curitiba. 2007. 139 f. Dissertação (Mestre em Engenharia de Produção e Sistemas) - Programa de Pós-Graduação em Engenharia de Produção e Sistemas, Pontifícia Universidade Católica do Paraná, Curitiba, 2007.

STOCK, J. R. Reverse logistics in the supply chain. Transport and logistics. June, 2001. 